GEOPHYSICAL RESEARCH LETTERS, VOL. 20, NO. 16, PAGES 1671-1674, AUGUST 20, 1993

\title{
THE EFFECT OF OXIDATION ON THE VERWEY TRANSITION IN MAGNETITE
}

\author{
Özden Özdemir and David J. Dunlop \\ Department of Physics, Erindale College, University of Toronto \\ Bruce M. Moskowitz \\ Department of Geology and Geophysics and Institute for Rock Magnetism, University of Minnesota
}

\begin{abstract}
At the Verwey transition $\left(\mathrm{T}_{\mathrm{V}} \approx 110-120 \mathrm{~K}\right)$, magnetite transforms from monoclinic to cubic spinel structure. It has long been believed that magnetic remanence and susceptibility would change markedly at $T_{V}$ in the case of coarse grains but only slightly or inappreciably in the case of fine $(<1 \mu \mathrm{m})$ grains. We find on the contrary that remanence changes at $T_{V}$ by $50-80 \%$ in both large and small crystals, if they are stoichiometric. However, minor surface oxidation suppresses the transition, and the fact that fine grains oxidize more readily leads to an apparent size dependence. Our experiments used submicron magnetite cubes with mean sizes of $0.037,0.076,0.10$ and $0.22 \mu \mathrm{m}$ which were initially non-stoichiometric (oxidation parameter $\mathrm{z}$ from 0.2-0.7). A saturation isothermal remanent magnetization (SIRM) given in a $2.5 \mathrm{~T}$ field at $5 \mathrm{~K}$ decreased steadily during zero-field warming to $300 \mathrm{~K}$ with little or no indication of the Verwey transition. After the oxidized surface of each crystal was reduced to stoichiometric magnetite, the SIRM decreased sharply during warming by $50-80 \%$ around $110 \mathrm{~K}$. The change in SIRM for the 0.22 $\mu \mathrm{m}$ grains was almost identical to that measured for a 1.5 $\mathrm{mm}$ natural magnetite crystal. Thus a $10^{12}$ change in particle volume does not materially affect the remanence transition at $T_{V}$ but oxidation to $z=0.3$ essentially suppresses the transition. The effect of the degree of oxidation on $\mathrm{T}_{\mathrm{V}}$ provides a sensitive test for maghemitization in soils, sediments and rocks.
\end{abstract}

\section{Introduction}

Magnetite exhibits a crystallographic phase transition from cubic to monoclinic at $110-120 \mathrm{~K}$, called the Verwey transition [Verwey, 1939; Zuo et al., 1990]. Associated with the transition is a magnetic isotropic point where the first magnetocrystalline anisotropy constant $K_{1}$ equals zero and the easy directions of magnetization change their orientation. The Verwey transition $\left(T_{v}\right)$ is a signature of magnetite and has been used as a technique for detecting magnetite in rocks. The transition is accompanied by a specific heat anomaly and abrupt changes in coercivity, remanence, susceptibility [Aragón et al., 1985; Aragón, 1992], and electrical conductivity [Verwey, 1939]. Although the details are still debated, some sort of electronic ordering occurs at the Verwey transition. Above $T_{V}$, rapid electron hopping occurs between randomly arranged octahedral ferrous and ferric ions in the cubic spinel lattice, but below $T_{V}$, electron hopping is inhibited due to ordering of the ferrous/ferric ions in a monoclinic structure, resulting in a 100-fold drop in conductivity [Verwey et al., 1947].

\section{Copyright 1993 by the American Geophysical Union.}

Paper number 93GL01483

0094-8534/93/93GL-01483\$03.00
Any deviation from stoichiometry in magnetite influences $T_{V}$ [e.g., Kuipers and Brabers, 1976]. Singlecrystal studies indicate that $T_{V}=118 \mathrm{~K}$ is a maximum for pure stoichiometric magnetite, but small degrees of hightemperature $(\mathrm{HT})$ nonstoichiometry $(\mathrm{z}<0.1)$ or impurities can depress the transition temperature below $100 \mathrm{~K}$ [e.g., Aragón et al., 1985]. When static remanence measurements are used to detect the Verwey transition, ultrafine grains exhibiting superparamagnetism can suppress the transition entirely due to the progressive unblocking of magnetization with temperature [e.g., Moskowitz et al., 1989].

In the present study, the effect of nonstoichiometry on the Verwey transition temperature is studied for magnetite crystals with mean sizes ranging from $0.04 \mu \mathrm{m}$ to 0.22 $\mu \mathrm{m}$. We also studied a $1.5 \mathrm{~mm}$ natural octahedral crystal of magnetite. The nonstoichiometry in the submicron magnetites is produced by low-temperature (LT) oxidation. Unlike HT nonstoichiometry $(z<0.1)$, much larger degrees of oxidation can be achieved at low temperatures ultimately resulting in the formation of maghemite $(z=1)$. The effects of LT oxidation on the Verwey transition have not been well documented.

In nature, the oxidation of magnetite to maghemite is probably the most common oxide mineral alteration. This reaction also invariably takes place in fine particles after exposure to air at room temperature [Topsøe et al., 1974]. Our experimental data are useful in establishing the remanence change at the Verwey transition as a sensitive probe for detecting low-temperature oxidation of magnetite in rocks, sediments, and soils.

\section{Experimental Procedure}

The magnetite samples are nearly perfect single crystal cubes grown from aqueous solution [Dunlop, 1973, 1986]. Median particle sizes were $0.22,0.10,0.076$ and $0.037 \mu \mathrm{m}$ for samples $1,2,3$ and 4 respectively (Table 1 ). The magnetites in their virgin state are surface oxidized to $\mathrm{\gamma Fe}_{2} \mathrm{O}_{3}$. We produced nearly stoichiometric magnetites by heating the magnetite samples in a mixture of $80 \% \mathrm{CO}_{2}$ and $20 \% \mathrm{CO}$ at $395^{\circ} \mathrm{C}$ for $146 \mathrm{hr}$.

No significant changes in grain size resulted from heating. For the $0.037 \mu \mathrm{m}$ grains, the saturation remanence ratio $M_{r s} / M_{s}$ was 0.24 initially and 0.21 after $146 \mathrm{hr}$ heating. The remanent coercivity ratio $\mathrm{H}_{\mathrm{cr}} / \mathrm{H}_{\mathrm{c}}$ changed even less: from 1.98 to 2.00 .

Remanence measurements were carried out on $2 \%$ by weight dispersions of oxide in a $\mathrm{CaF}_{2}$ matrix. Samples were hydraulically pressed into small cylindrical pellets. The natural single crystal of magnetite is $1.5 \mathrm{~mm}$ in size and comes from the Nordmark mine, Sweden. The maghemite particles, manufactured by Pfizer, were acicular (length $0.45 \mu \mathrm{m}$, axial ratio 5:1).

Low-temperature remanence measurements were made with a superconducting susceptometer whose residual field is $<300 \mu \mathrm{T}$. Hysteresis curves were measured at room temperature in a maximum field of $1 \mathrm{~T}$ using a vibrating 
Table 1. Properties of stoichiometric and non-stoichiometric magnetites.

\begin{tabular}{|c|c|c|c|c|c|c|c|c|}
\hline Sample & $\begin{array}{c}\mathrm{d} \\
\mu \mathrm{m}\end{array}$ & $\begin{array}{l}\mathbf{a} \\
\AA\end{array}$ & $\begin{array}{c}\mathrm{a}_{\mathrm{ox}} \\
\AA\end{array}$ & $x$ & $\begin{array}{c}\mathrm{M}_{\mathrm{s}} \\
\mathrm{Am}^{2} / \mathrm{kg}\end{array}$ & $\begin{array}{c}\mathrm{M}_{\mathrm{Sox}} \\
\mathrm{Am}^{2} / \mathrm{kg}\end{array}$ & $R_{1}$ & $\mathrm{R}_{\mathrm{I} \text { ox }}$ \\
\hline 1 & 0.22 & $8.400 \pm 0.005$ & $8.392 \pm 0.008$ & 0.20 & 88.3 & 85.8 & 0.231 & 0.326 \\
\hline 2 & 0.10 & $8.399 \pm 0.005$ & $8.389 \pm 0.004$ & 0.32 & 88.2 & 84.5 & 0.287 & 0.369 \\
\hline 3 & 0.076 & $8.398 \pm 0.004$ & $8.386 \pm 0.007$ & 0.64 & 83.0 & 77.3 & 0.303 & 0.424 \\
\hline 4 & 0.037 & $8.393 \pm 0.008$ & $8.376 \pm 0.004$ & 0.73 & 82.5 & 69.6 & 0.372 & 0.496 \\
\hline $\begin{array}{l}\text { Natural } \\
\text { single } \\
\text { crystal }\end{array}$ & 1500 & $8.397 \pm 0.002$ & & & 88.3 & & 0.090 & \\
\hline Maghemite & 0.45 & 8.34 & & & 75.5 & & & \\
\hline
\end{tabular}

$d$ is particle size; $a$ and $a_{o x}$ are spinel cell-edge parameters for the reduced and oxidized magnetites, respectively; $M_{s}$ and $M_{s o x}$ are saturation magnetizations; $R_{I}$ and $R_{I}$ are the SIRM memory fractions; $z$ is the oxidation parameter.

sample magnetometer. X-ray powder pictures of the samples were taken with a Guiner de Wolff camera using $\mathrm{Cu}-\mathrm{K} \alpha$ radiation and high purity silicon as an internal standard (NBS standard SRM-640).

\section{Experimental Results}

\section{Sample Characterization}

The X-ray cell-edge parameters for the surface oxidized magnetites (Table 1) are in the range 8.376-8.392 $\AA$. The values of oxidation parameter, $z$, listed in Table 1 were obtained by comparing our cell-edge data with the calibration curve of Readman and O'Reilly [1972]. The $z$ values of the non-stoichiometric samples increase with decreasing particle size, from 0.20 for the largest crystals $(0.22 \mu \mathrm{m})$ to 0.73 for the finest ones $(0.037 \mu \mathrm{m})$. The smaller particles have larger surface/volume ratios and oxidize more readily. The dependence of oxidation on particle size was also reflected in saturation magnetization data. $\mathrm{M}_{\mathrm{s}}$ decreased from 85.8 to $69.6 \mathrm{Am}^{2} / \mathrm{kg}$ as the particle size decreased from 0.22 to $0.037 \mu \mathrm{m}$.

After reduction, the magnetites were nearly stoichiometric and had cell-edge parameters of 8.393-8.400 $\AA$. The saturation magnetization values also increased: from 69.6 to $83 \mathrm{Am}^{2} / \mathrm{kg}$ for sample 4 and from 85.8 to 88.3 for sample 1 . These increases in $\mathrm{M}_{\mathrm{s}}$ and X-ray parameter indicate that the maghemitized surface layer was reduced to nearly stoichiometric $\mathrm{Fe}_{3} \mathrm{O}_{4}$ after long periods of heat treatment in $\mathrm{CO} / \mathrm{CO}_{2}$.

The natural octahedral magnetite crystal was analysed using an SEM/X-ray microanalyser. A small amount of manganese impurity is present.

\section{Low-temperature remanence measurements}

The surface oxidized and reduced magnetites were each given a saturation isothermal remanent magnetization (SIRM) in a field of $2.5 \mathrm{~T}$ at a temperature of $5 \mathrm{~K}$, then warmed to $300 \mathrm{~K}$ in approximately zero field (Figure 1). We also measured the low temperature memory by a final cooling step back to $5 \mathrm{~K}$ in zero field. The ratios $R_{1}$ and $\mathbf{R}_{\text {Iox }}$ of this surviving SIRM after zero-field cycling from 5 $\mathrm{K}$ to $300 \mathrm{~K}$ and back to $5 \mathrm{~K}$ to the original SIRM induced in $2.5 \mathrm{~T}$ at $5 \mathrm{~K}$ for the stoichiometric and nonstoichiometric magnetites are given in Table 1 .

On warming, the SIRM of the oxidized magnetites decreased rapidly between 5 and $40 \mathrm{~K}$. SIRM changed very little between 40 and $80 \mathrm{~K}$, then decreased slowly for samples 2,3 and $4(0.037,0.076$ and $0.10 \mu \mathrm{m})$. There
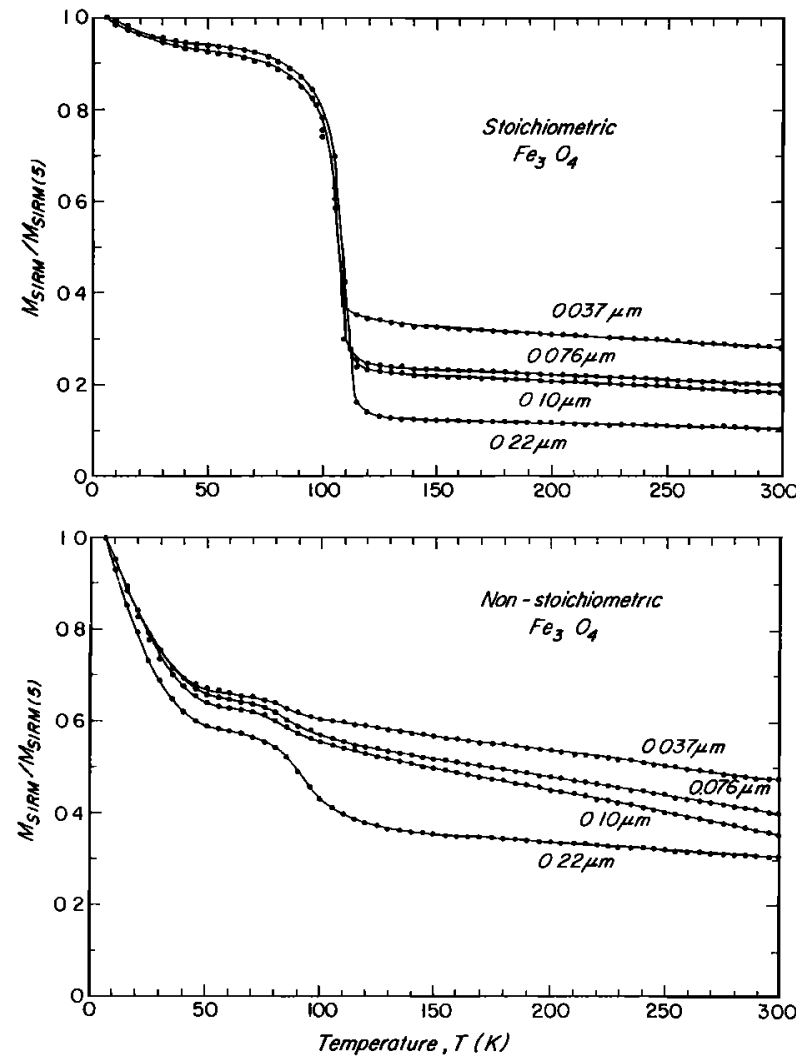

Fig. 1. Normalized saturation isothermal remanence (SIRM) curves of magnetites during warming from $5 \mathrm{~K}$ to $300 \mathrm{~K}$ in zero field. Top: reduced magnetites. Below the Verwey transition the remanence curves are almost identical. Bottom: surface oxidized magnetites. The Verwey transition is suppressed as a result of oxidation.

was only a slight indication of the Verwey transition for these three samples. The $0.22 \mu \mathrm{m}$ non-stoichiometric magnetite does undergo a transition, but at a lower temperature than in stoichiometric magnetite. For this series, the transitions are not sharp but are spread over a temperature interval of at least $30 \mathrm{~K}$.

When the oxidized surface layer of $\gamma-\mathrm{Fe}_{2} \mathrm{O}_{3}$ was reduced to pure $\mathrm{Fe}_{3} \mathrm{O}_{4}$, a sharp decrease in SIRM around $110 \mathrm{~K}$ was observed on the same submicron magnetites. The shapes of the remanence curves between 5 and $110 \mathrm{~K}$ and also the Verwey transition temperatures $\left(T_{V}\right)$ were the 
same for all the stoichiometric magnetites, independent of grain size. However the amount of remanence lost at $T_{V}$ increases with increasing grain size.

The low-temperature remanence curve of the natural single crystal of magnetite (Figure 2 ) is very similar to that of the $0.22 \mu \mathrm{m}$ stoichiometric magnetite. The Verwey transition occurred at $T_{V}=102 \mathrm{~K}$, lower than in the synthetic stoichiometric magnetites, probably because of the manganese impurity or slight HT nonstoichiometry [Aragón et al., 1985]. The initial drop in remanence below $20 \mathrm{~K}$ is intriguing but its cause is unknown.

\section{Discussion}

From static remanence measurements, we have found that the Verwey transition occurs in near stoichiometric magnetite crystals for all sizes above $37 \mathrm{~nm}$. Below this size, the effects of superparamagnetism become significant and the detection of $T_{V}$ by remanence measurements is made difficult. Moskowitz et al. [1989] found that magnetite with mean particle size of $10 \mathrm{~nm}$, produced by dissimilatory iron-reducing bacteria, shows no evidence of a Verwey transition using either remanent magnetization or Mössbauer methods. Besides superparamagnetism, the Verwey transition is significantly influenced by nonstoichiometry. With increasing LT oxidation, the transition becomes blurred, shifts to lower temperatures, and eventually disappears for pure maghemite (Figures 1 and 2).

During the oxidation of magnetite, $\mathrm{Fe}^{2+}$ ions diffuse to the surface of the crystal and oxidize to $\mathrm{Fe}^{3+}$, leaving lattice vacancies in octahedral sites [Gallagher et al., 1968]. As the number of $\mathrm{Fe}^{2+}$ ions decreases, electron hopping between $\mathrm{Fe}^{2+}-\mathrm{Fe}^{3+}$ octahedral pairs is inhibited [Daniels and Rosencwaig, 1969]. In pure $\gamma-\mathrm{Fe}_{2} \mathrm{O}_{3}$, octahedral sites are completely occupied by $\mathrm{Fe}^{3+}$ ions and vacancies and no electron hopping can occur. Figure 2 shows the temperature dependence of remanence for the acicular $\gamma-\mathrm{Fe}_{2} \mathrm{O}_{3}$ particles. There is a gradual decrease of the SIRM between 5 and $300 \mathrm{~K}$ but no Verwey transition.

In their virgin state, the fine magnetite particles used in the present study consist of a fully oxidized surface shell of $\mathrm{Fe}_{2} \mathrm{O}_{3}$ and a core of essentially unoxidized $\mathrm{Fe}_{3} \mathrm{O}_{4}$, separated by a transitional region with a gradient in z [Feitknecht and Gallagher, 1970; Ózdemir and Dunlop, 1989]. If the magnetite core and its surrounding region of only slightly cation-deficient magnetite are large enough, electron hopping will still occur and a Verwey transition

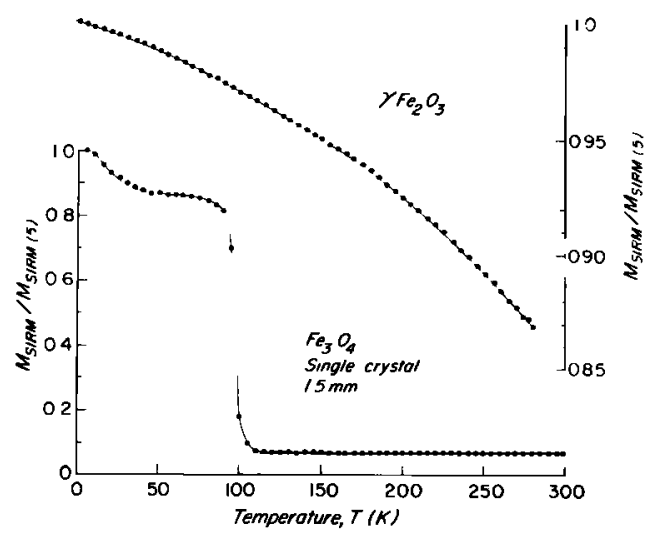

Fig. 2. Normalized SIRM curves of acicular maghemite particles $(0.45 \mu \mathrm{m}$ length) and a natural single crystal of magnetite (1.5 mm diameter) during warming to $300 \mathrm{~K}$ in zero field. will be observed, although spread out over a range of temperatures because of the spread of $z$ values. This is the situation in the relatively large crystals of sample 1 (Figure 1), where the transition occurs over the range 70-120 K. In the finer crystals, the average oxidation state is so high that electron hopping is suppressed, and the Verwey transition almost disappears (samples 2, 3 and 4, Figure 1).

Between 5 and $40 \mathrm{~K}$, the low-temperature remanence of all the oxidized magnetites decreases by $30-40 \%$ (Figure 1). This phenomenon is due to superparamagnetism. Because oxidation is not uniform throughout the crystal, the oxidized surface shell, with a smaller lattice parameter than the unoxidized magnetite core, stretches and cracks. The outer surface of fine particles with a strong oxidation gradient then resembles a cluster of closely spaced superparamagnetic (SP) and nearly SPsize particles. The nearly SP particles will have very low SIRM unblocking temperatures. When the partially oxidized magnetites were reduced to their stoichiometric compositions, the strong decrease in SIRM between 5 and $40 \mathrm{~K}$ disappeared (Figure 1), presumably because the cracked surface healed during 6 days' annealing at $395^{\circ} \mathrm{C}$.

The magnetic memory ratios, $R_{I}$, for both stoichiometric and nonstoichiometric magnetites increased with decreasing particle size (Table 1). Similar results for SIRM and other remanences acquired at room temperature and cooled through $T_{V}$ have been reported for crushed and grown magnetites [Dunlop and Argyle, 1991; Heider et al., 1992]. In our experiments, the memory was greater for partially oxidized grains than for unoxidized grains of the same size. Enhanced magnetic memory in these nonstoichiometric magnetites must be controlled by the residual stress developed during oxidation. Values of the room-temperature coercive force, $\mathrm{H}_{c}$, of the nonstoichiometric magnetites are substantially higher than those of the reduced magnetites, and have a stronger dependence on grain size (Figure 3). Both trends are consistent with higher residual stresses in the oxidized material [Dunlop, 1986]. The stress is a direct result of the oxidation gradient and resulting variation in lattice parameter in nonstoichiometric grains.

\section{Implications for Environmental Magnetism}

The detailed rock magnetic record of the loess/paleosol sequences of NW and central China offers a potentially high-resolution record of paleoclimatic and paleoenvironmental change spanning the Quaternary period

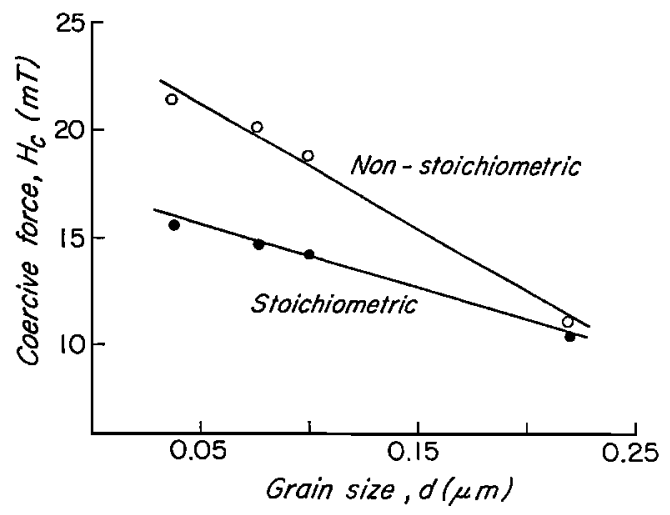

Fig. 3. Coencive force as a function of particle size for the surface oxidized and reduced magnetites. Coercive forces of partially oxidized magnetites are higher than those of monophase magnetites because of the stress developed at the $\gamma \mathrm{Fe}_{2} \mathrm{O}_{3}-\mathrm{Fe}_{3} \mathrm{O}_{4}$ interface during oxidation. 
[e.g., Wang et al., 1990]. Recent studies suggest that during periods of higher temperatures and rainfall, pedogenic processes produce SP and SD particles of magnetite, maghemite, or partially oxidized magnetite [e.g., Singer and Fine, 1989; Banerjee et al., 1993]. However, the positive identification of maghemite in ultrafine particles is not easy. Thermomagnetic analyses are complicated by mineralogical changes occurring in both oxides and clay minerals during heating. Mössbauer spectroscopy is made difficult by the presence of ironbearing clay minerals and SP particles, as well as very low concentrations of magnetic minerals.

Monitoring the changes in SIRM at low temperatures, as we have done in our experiments, is an attractive alternative for detecting maghemitization. If $S D$ or $M D$ magnetite is present, it should be recognizable from its remanence transition. A muted remanence transition, spread over a broad temperature range, suggests lowtemperature oxidation. With sufficient oxidation, the transition will be suppressed entirely. SP and near-SP surface oxidized particles are indicated by a strong decrease in SIRM just above $5 \mathrm{~K}$. However, it may be difficult to distinguish the above case from a mixture of SP and non-SP particles of pure stoichiometric magnetite because both cases will produce similar SIRM temperature responses. A possible solution to this problem is the observation that LT nonstoichiometry smears out the transition and shifts $T_{V}$ to lower temperatures. Although much work remains to be done to establish trends in the Verwey transition as quantitative indicators of the degree of oxidation, the potential for such a method is clearly indicated by our results.

\section{Conclusions}

1. A change in remanence occurs at the Verwey transition in stoichiometric magnetite of all sizes above the superparamagnetic threshold size. The remanence transition temperature was $110 \mathrm{~K}$ in magnetites having grain sizes between 0.037 and $0.22 \mu \mathrm{m}$. The natural $1.5 \mathrm{~mm}$ single crystal of magnetite had a similar transition at $102 \mathrm{~K}$.

2. Small deviations from stoichiometry in magnetite have a significant effect on the Verwey transition. This influence is dramatically illustrated by the spreading out and eventual suppression of the remanence transition in partially oxidized magnetites. This property can be used as a sensitive probe for detecting low-temperature oxidation in rocks, sediments and soils.

3. The magnetic memory of submicron magnetites increases with decreasing particle size. Partially oxidized magnetite particles have higher memory ratios than stoichiometric crystals. Magnetic memory is controlled in part by the internal stresses developed at the $\gamma \mathrm{Fe}_{2} \mathrm{O}_{3}-\mathrm{Fe}_{3} \mathrm{O}_{4}$ interface during oxidation. These stresses also impart higher coercivities to partially oxidized crystals.

Acknowledgments. We thank Subir Banerjee, Jim Marvin and Chris Hunt of the Institute for Rock Magnetism for help with the experiments, Malcolm Back and Bob Ramik of the Royal Ontario Museum, Toronto for donating the natural single crystal of magnetite, Mark Ward of the Department of Metallurgy and Materials Science, University of Toronto for reducing our magnetites, and Carolyn Moon for typing the manuscript. This research was supported by NSERC Operating Grant A7709 to D.J.D. This is contribution 9302 of the Institute for Rock Magnetism. Support for the IRM is provided by grants from the Keck Foundation and the National Science Foundation.

\section{References}

Aragón, R., et al., Influence of nonstoichiometry on the Verwey transition, Phys. Rev., 31, 430-436, 1985.

Aragón, R., Magnetization and exchange in nonstoichiometric magnetite, Phys. Rev., 46, 53285333, 1992.

Banerjee, S.K., et al., Separation of local signals from the regional paleomonsoon record of the Chinese loess plateau: A rock-magnetic approach, Geophys. Res. Lett., in press, 1993.

Daniels, J.M., and A. Rosencwaig, Mössbauer spectroscopy of stoichiometric and non-stoichiometric magnetite, J. Phys. Chem. Solids, 30, 1561-1571, 1969.

Dunlop, D.J., Superparamagnetic and single domain

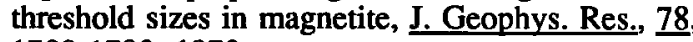
1780-1793, 1973.

Dunlop, D.J., Hysteresis properties of magnetite and their dependence on particle size: A test of pseudo-singledomain remanence models, J. Geophys. Res., 91, 95699584, 1986.

Dunlop, D.J., and K.S. Argyle, Separating multidomain and single-domain-like remanences in pseudo-singledomain magnetites (215-540 $\mathrm{nm}$ ) by low-temperature demagnetization, J. Geophys. Res., 96, 2007-2017,1991.

Feitknecht, W., and K.J. Gallagher, Mechanisms for the oxidation of $\mathrm{Fe}_{3} \mathrm{O}_{4}$, Nature, 228, 548-549, 1970.

Gallagher, K.J., et al., Mechanism of oxidation of magnetite to $\gamma-\mathrm{Fe}_{2} \mathrm{O}_{3}$, Nature, 217, 1118-1121, 1968.

Heider, F., et al., Low-temperature and alternating field demagnetization of saturation remanence and thermoremanence in magnetite grains $(0.037 \mu \mathrm{m}$ to 5 mm), J. Geophys. Res., 97, 9371-9381, 1992.

Kuipers, A.J.M., and V.A.M. Brabers, Thermoelectric properties of magnetite at the Verwey transition, Phys. Rev., 14, 1401-1405, 1976.

Moskowitz, B.M., et al., A comparison of magnetite particles produced anaerobically by magnetotactic and dissimilatory iron-reducing bacteria, Geophys. Res. Lett., 16, 665-668, 1989.

Özdemir, Ö., and D.J. Dunlop, Chemico-viscous remanent magnetization in the $\mathrm{Fe}_{3} \mathrm{O}_{4}-\gamma \mathrm{Fe}_{2} \mathrm{O}_{3}$ system, $\underline{\text { Science, }}$ 243, 1043-1047, 1989.

Readman, P.W., and W. O'Reilly, Magnetic properties of oxidized (cation-deficient) titanomagnetites $(\mathrm{Fe}, \mathrm{Ti}$, $\left.\square_{3}\right) \mathrm{O}_{4}$, J. Geomag. Geoelectr., 24, 69-90, 1972.

Singer, M.J., and P. Fine, Pedogenic factors affecting magnetic susceptibility of northern California soils, Soil Sci. Soc. Am. J., 53, 1119-1127, 1989.

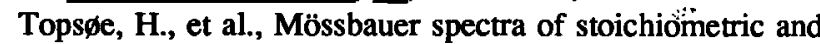
nonstoichiometric $\mathrm{Fe}_{3} \mathrm{O}_{4}$ microcrystals, J. de Physique, 35, 411-413, 1974.

Verwey, E.J.W., Electronic conduction of magnetite $\left(\mathrm{Fe}_{3} \mathrm{O}_{4}\right)$ and its transition point at low temperatures, Nature, 144, 327-328, 1939.

Verwey, E.J., et al., Physical properties and cation arrangement of oxides with spinel structures, J. Chem. Phys, 15, 181-187, 1947.

Wang, Y.M., et al., Magnetic susceptibility of Chinese loess and its bearing on paleoclimate, Geophys. Res. Lett., 17, 2449-2451, 1990.

Zuo, J.M., et al., Charge ordering in magnetite at low temperatures, Phys. Rev., 42, 8451-8464, 1990.

Ö. Özdemir and D.J. Dunlop, Department of Physics,

Erindale College, University of Toronto, Mississauga,

Ontario, Canada L5L 1C6.

B.M. Moskowitz, Department of Geology and

Geophysics and Institute for Rock Magnetism, University of Minnesota, Minneapolis, MN 55455, U.S.A.

(Received March 9, 1993; accepted May 12, 1993.) 